Cahiers de Narratologie

Analyse et théorie narratives

Avant-gardes et littérature narrative

\title{
Les projets romanesques de F.T. Marinetti : (im)précis de génétique textuelle
}

Barbara Meazzi

\section{(2) OpenEdition}

Journals

Édition électronique

URL : http://journals.openedition.org/narratologie/6725

DOI : 10.4000/narratologie.6725

ISSN : $1765-307 X$

Éditeur

LIRCES

Référence électronique

Barbara Meazzi, «Les projets romanesques de F.T. Marinetti : (im)précis de génétique textuelle», Cahiers de Narratologie [En ligne], 24 | 2013, mis en ligne le 17 septembre 2013, consulté le 01 mai 2019. URL : http://journals.openedition.org/narratologie/6725; DOI : 10.4000/narratologie.6725

Ce document a été généré automatiquement le 1 mai 2019.

Article L.111-1 du Code de la propriété intellectuelle. 


\title{
Les projets romanesques de F.T. Marinetti : (im)précis de génétique
} textuelle

\author{
Barbara Meazzi
}

«Penso a un romanzo Luce di donna o Chiaro di donna.

Dopo la guerra le forze mancano ${ }^{1}$ "

1 Les archives de Marinetti conservées à la Beinecke Library contiennent, entre autres, le journal qu'il rédigea à partir au moins de 1915 : une partie de ces textes fut publiée en 1987 chez Il Mulino par Alberto Bertoni² ${ }^{2}$. Cette première édition a permis d'appréhender de manière assez différente la personnalité de Marinetti, son Imaginaire, son écriture ainsi qu'une partie de ses faits et de ses gestes pendant la Première Guerre mondiale. Ce document très précieux contient néanmoins de nombreuses méprises, ce qui est compréhensible compte tenu de l'énorme travail de transcription que cela représente. Comme nous le verrons ici, bien plus gênantes pour l'appréhension de l'artiste, de sa personnalité, de son Imaginaire et de son écriture apparaissent, en revanche, les nombreuses omissions, d'autant plus qu'à aucun moment elles n'ont été signalées et qu'elles concernent essentiellement les aspects littéraires. De fait, tel qu'il a été publié, le volume est finalement surtout une précieuse égographie, un document historique rédigé par un forcené de la guerre, ce que Marinetti a été aussi, alors que sa personnalité de poète et d'homme de lettres demeure quelque peu secondaire.

2 En nous appuyant sur quelques exemples, nous montrerons que ces carnets, tout en contribuant à la reconstruction de l'histoire de l'avant-garde en Italie et en Europe, ont une valeur littéraire et poétique importante et sont, entre autre, un laboratoire d'expérimentation romanesque.

3 Pour des raisons évidentes de protection de la vie privée, dans la transcription des carnets n'apparaissent jamais les noms propres des personnes - surtout des femmes - que Marinetti fréquente à cette époque. Tous les noms, les prénoms et les adresses ont été 
systématiquement remplacés par des initiales, lit-on dans la note introductive : cela se justifie pour les personnes " inconnues ", mais pour les artistes et pour les écrivains cette lacune est regrettable, parce que cela nous aurait permis d'avoir une idée encore plus précise de l'éclectisme et de l'agitation perpétuelle du poète, en tout comparables à ceux d'autres poètes et artistes-soldats qui ne cessent d'alimenter leurs correspondances pour s'accrocher à la normalité de la vie loin de la guerre.

4 En comparant le manuscrit avec l'édition publiée, parmi les initiales nous retrouvons par exemple le nom de Mina Della Pergola ${ }^{3}$, jeune futuriste maîtresse de Marinetti pendant l'été 1918, ou bien celui d'Hélène d'ÆEttingen, la cousine de Serge Férat chez qui, en 1913, Apollinaire, Boccioni et Marinetti avaient conçu le «Manifeste de l'Antitradition futuriste ». Est-ce qu'Hélène d'CEttingen eut une aventure avec le fondateur du futurisme? De toute évidence cela n'a pas de grande importance parce que ce qui compte, incontestablement, c'est de savoir si certaines relations ont abouti à une coopération artistique et intellectuelle, comme ce fut le cas par exemple avec Enif Robert ${ }^{4}$ : le roman Un ventre di donna publié en 1919 chez Facchi et écrit à quatre mains fut justement le fruit d'un rapport intense, au moins intellectuellement.

5 Il est tout de même possible, du reste, que les ardeurs du Casanova du futurisme aient épargné certaines femmes dont les noms sont pourtant passés sous silence dans cette édition: c'est probablement ce qu'il advint avec Hélène d'Ættingen, par exemple, qui apparaît dans une liste, hélas non datée, à côté de l'indication de l'adresse (229 bd Raspail), sous l'annotation "voir à Paris » (vedere a Parigi). La correspondance connue d'Hélène et celle de Marinetti ne permettent pas, pour l'heure, de connaitre l'état exact de leurs relations pendant la guerre, néanmoins il est tout à fait possible que Marinetti ait servi d'intermédiaire entre la Baronne et Mario Brosio, le directeur de la revue Valori Plastici, au début des années vingt: cela expliquerait pourquoi Roch Grey publia un ouvrage sur Henri Rousseau et un sur Van Gogh chez "Valori Plastici »-et Crès -, respectivement en 1921 et en 1924.

D'autres lacunes, dans les Taccuini, sont en revanche bien plus lourdes de conséquences pour les spécialistes de la matière futuriste. Un exemple parmi d'autres : de temps à autre apparaissent des points de suspension entre crochets, "[...]», ce qui indique normalement des coupures dans le texte. Au nom de l'usage conventionnel, par conséquent, il nous est arrivé d'émettre des hypothèses qui se sont révélées fausses par la suite $^{5}$, après vérification dans le manuscrit, tout simplement parce que l'éditeur a eu recours aux crochets surtout pour signaler les mots ou les parties illisibles, et il est vrai que la qualité du document est dans l'ensemble assez inégale. Parfois les crochets sont utilisés correctement; parfois, en revanche, les coupures ne sont pas du tout signalées, comme dans l'exemple suivant:

Enif Robert. Âme inquiète. Âme ultra nerveuse. «J'aime me peindre de méchanceté.

Ensuite je me repens ${ }^{6}$."

C'est ainsi qu'est transcrit le texte, tandis que la version manuscrite est plus longue :

Enif Robert. Âme inquiète. Âme ultra nerveuse. «J'aime me peindre de méchanceté. Ensuite je me repens. Aujourd'hui tu ne m'aimes pas. Une ombre est descendue entre nous. Pourquoi [ill.]» Enif me dit ces paroles étranges [ill.] au lit [ill ${ }^{7}$.]

8 Marinetti était au lit avec Enif Robert: voilà un scoop qui nous laisse parfaitement indifférents et qui ne bouleversera guère les études sur le futurisme ${ }^{8}$. Encore faut-il être certains que tout ce qu'écrit Marinetti dans ses carnets est réel - notamment les récits de ses performances sexuelles - et que cela ait un quelconque intérêt littéraire. 
D'autres éléments ont été omis sans explications et sans indications, comme par exemple les «notes de précisions linguistiques", à savoir des listes de noms ou d'expressions, rédigées surtout en italien. Voici une amusante petite liste de «bruits de baisers » :

Rumorismo di baci [Bruitisme de baisers]

mmzzz

[ill.]

mpsc

mpds 9

10 Ces notes, assez nombreuses, indiquent les hésitations de l'auteur quant à l'utilisation de la langue italienne et leur prolifération nous donne des indications importantes sur la méthode de travail du poète Marinetti qui, au moins depuis l'époque de Mafarka le futuriste (1909), tire de là son inspiration :

Affinité / antivoyance / effusions de bonté / faire une grimace avec la bouche pour commencer à pleurer / toutes les bassesses le révulsent / satisfaire sa soif / veste gilet / il me suivait dans les escaliers tout doucement me tenir à la rambarde / Je t'ai vu bras dessus bras dessous avec une ivrogne / une longue table autour de laquelle étaient assis tous ces hommes / je tombai à genoux / marchant le long du parapet / une lumière douce tamisée [...] (se) poursuit sans fin ${ }^{10}$

Parmi ces "notes de précisions linguistiques », assez nombreuses et de toutes sortes, on retrouve des listes organisées par champs sémantiques, par exemple les sous-vêtements élégants pour dame, ou bien des séries d'images assez saisissantes, pouvant être lues comme des vers d'un poème inachevé, entrecoupés par les bruits des bombardements. Presque de l'écriture automatique :

Dès que la nuit tombe, je reste à l'écoute / Il souffrait d'engelures / Plaies aux pieds saignants / Attacher ses chaussures / Je sentais ses mains sur mes épaules pendant qu'à genoux je léchais sa chatte / Je m'attardais et ses mains tremblaient / Combien de jours lui donnez-vous à en juger par son aspect? / Je sentais sur ma bouche l'impression de tes paupières douces comme deux altos / J'irai droit jusqu'au bout / Où étions-nous ? / À table j'étais presque contre lui / Il dépassait tout le monde / Il ne craignait pas de se contredire / Répulsion et attraction / À la fin du dîner / Il se fâchait facilement / Il tenait tête à tout le monde / Le soleil, peut-être enhardi par le vin de toutes les tavernes pillées par le sang [ill.] tenait tête à la nuit ${ }^{11}$.

Il convient cependant de souligner que la partie des carnets manuscrits que nous avons consultée ne contient que peu de planches motlibristes par rapport à l'abondance des projets romanesques. Cela est assez curieux, étant donné que Marinetti publie beaucoup de mots en liberté dans L'Italia futurista. De plus, et à plusieurs reprises, il lui arrive de faire allusion à un projet anthologique qui ne verra cependant pas le jour - de toute manière les parties des carnets concernant ce projet n'ont pas été transcrites. En vérité, et si l'on veut être précis, les planches dessinées dans les carnets ne ressemblent pas tout à fait à celles que Marinetti publie dans les revues, vraisemblablement parce que Marinetti les travaille ailleurs que dans les carnets.

Puis il y a les projets de romans, dont A. Bertoni rend compte, en se limitant la plupart des fois à signaler les titres, alors que très souvent Marinetti développe aussi l'intrigue. En vérité, ce qui ressort, c'est que l'imagination et l'Imaginaire du fondateur du futurisme débordent de projets et des projets romanesques, ce qui ne préjuge en rien de leur réalisation. Et même, les titres et les récits qui en découlent sont si nombreux qu'il devient légitime de se demander non pas s'il a existé un roman futuriste, mais pourquoi à cette époque Marinetti n'a pas fait du roman le fer de lance du futurisme. 


\section{«Mitragliando e baciando » : éros et thanatos}

14 Avant toutefois de nous interroger sur cette question et d'en analyser le sens et les conséquences, il convient déjà de répertorier et d'examiner quelques projets romanesques contenus dans les carnets ${ }^{12}$.

15 Marinetti utilise les termes «roman » et «livre » de manière tout à fait équivalente : «Écrire un roman », « créer un livre », «je pense à un roman », «titres de livres ». En principe et pour l'heure un roman est un livre, tandis qu'un livre peut ne pas être un roman. La distinction ne va pas de soi, surtout si l'on considère le livre futuriste dans une perspective diachronique : dans les années trente, le « livre » futuriste se transformera, se réifiera et deviendra par exemple un objet - les Litolatte $^{13}$. Quant au roman - nous n'avons pas la prétention ici de parcourir l'histoire du roman futuriste - conçu à un moment donné comme une boîte (ce sera le cas pour Scatole d'amore in conserva, publié en 1927), il deviendra ensuite un disque, Amanti simultanei - romanzo discato ${ }^{14}$, donc un objet sonore.

Dès cette époque pourtant, Marinetti perçoit les limites expressives représentées par l'écriture romanesque: s'il s'exprime une fois en faveur de la mise à mort du roman traditionnel, il n'est pas certain qu'il ait pendant la guerre une idée très précise de ce qu'il faudrait faire pour écrire un roman non traditionnel. Songeait-il à ses lectures scapigliate de sa jeunesse et aux considérations exprimées par Dossi ou par Tarchetti ${ }^{15}$ ? Peut-être :

6789 avril [1918, n.d.r.] [...] Le roman doit mourir. À présent sa nostalgie est déplacée. D'Homère à Rosny et à D'Annunzio, le roman a toujours gardé son rythme nostalgique de récit désolé : il était une fois... Nous opposons à cette plainte-regret :

fais, choisis, défonce, domine comme ça, ça, et aussi ça... comme ça, ça ! et ça ${ }^{16}$ !

Malgré et au-delà de ces approximations, c'est pourtant dès 1918 qu'il conçoit Gli amori futuristi ${ }^{17}$ : ni livre, ni roman, ni tout à fait recueil de nouvelles, Gli amori futuristi seront des « [p]rogrammes de vie avec des ramifications. C'est-à-dire avec des variantes dans le final. 2 ou 3 conclusions possibles, différentes les unes des autres et au choix ${ }^{18}$ ».

$\mathrm{Au}$ fil des notes, nous apprenons que Marinetti imagine d'écrire un ou des romans synthétiques futuristes, sans donner plus de précisions; il songe à une "espèce de roman " ou bien à un " petit roman ", autant de signes indiquant l'inadéquation du genre par rapport à la réalité qu'il est en train de vivre. Au reste, il conçoit un roman « de vie vécue » ou bien un «roman vécu de guerre vécu». Il faut noter que, comme déjà les "programmes de vie», la formulation renvoie à une conception esthétique futuriste selon laquelle l'art et la vie coïncident. À partir de cette idée naîtra d'ailleurs L'alcova d'acciaio. Romanzo vissuto ${ }^{19}$, assez fidèlement extrait des carnets manuscrits.

En somme, Marinetti au front se doit de "dire le monde» sous toutes les formes possibles, à la fois par crânerie et par désespoir. Il imagine tout et son contraire, il annonce la mort du roman et imagine d'en écrire des dizaines. De même, il possède toutes les femmes mais ne peut en féconder aucune, se sachant suspendu entre la vie et la mort et condamné au provisoire, comme ses notes qui deviendront, peut-être, des « livres ». Il se décrit à la fois dans l'abjection du sexe payant et stérile, parce que la guerre est immanence dégradante, et sous la forme d'un nouveau Prométhée, capable de fertiliser le monde par son œuvre :

Moi, ici, je n'ai que de la boue... Mais, comme Dieu modela l'homme avec la boue,

j'espère à mon tour pétrir le nouveau monde futuriste italien ${ }^{20}$. 
poétiques et romanesques à l'état brut :

J'organise mon roman L'Interventionniste ou Contre le dépôt du clair de lune ou L'Amant rapide. Frénésie des trains entre Milan et Rome Naples. Manifestation interventionniste au théâtre Dal Verme en Galleria. 8 drapeaux autrichiens brûlés. Quelques arrêts brefs à Rapallo Golfe de douceur érotique à jambes ouvertes. Un autre roman : La Lettre charnelle ou La Lettre de chair. (21 mars 191721)

21 Et encore, plus loin :

$22 \mathrm{Au}$ lit dans la réserve de Moraro. Bronchite.

Dans le roman L'Amant rapide ou L'Interventionniste décrire largement et de façon convaincante les nuits tourmentées des batailles lointaines, les nuits de luxure déchirées envahies ravagées par les hordes russes criantes, par les bombardements. (24 mars 191722)

Une année plus tard et après avoir vu la mort de très près, son attitude demeure inchangée :

Je veux écrire des mots en liberté

Le Paysage d'odeurs de mon chien

Les Odeurs de mon Amie

Avec les mots en liberté décrire un coït passionné.

(Titres de livres Luxure de guerre Mes nuits Madame [ill.] Pages torrides Nuits torrides Lit champ de bataille Sac de couchage Les Problèmes de mon corps Bordel de guerre à côté des tranchées.) (18 avril 1918²3)

Les projets de romans type Mitragliando e baciando (mitraillant et embrassant) ou Sacco a pelo (sac de couchage) ne sont pas explicités par Marinetti : vu les titres, ce n'est peut-être pas une grande perte pour la littérature.

Apparaissent cependant aussi d'autres canevas de projets littéraires: c'est le cas de l'intrigue du roman Deux lettres d'amour se rencontrent, rédigée en partie en italien et en partie en français le 10 avril 1917. Ce projet pourrait tout à fait se rapprocher de la planche "Le soir couchée dans son lit / Elle relisait la lettre de son artilleur au front", parue dans L'Italia futurista le 9 septembre 1917 sous forme de publicité motlibriste, et que Marinetti publiera à nouveau dans Les Mots en liberté futuristes en 1919, après avoir ôté la partie supérieure de l'original. 


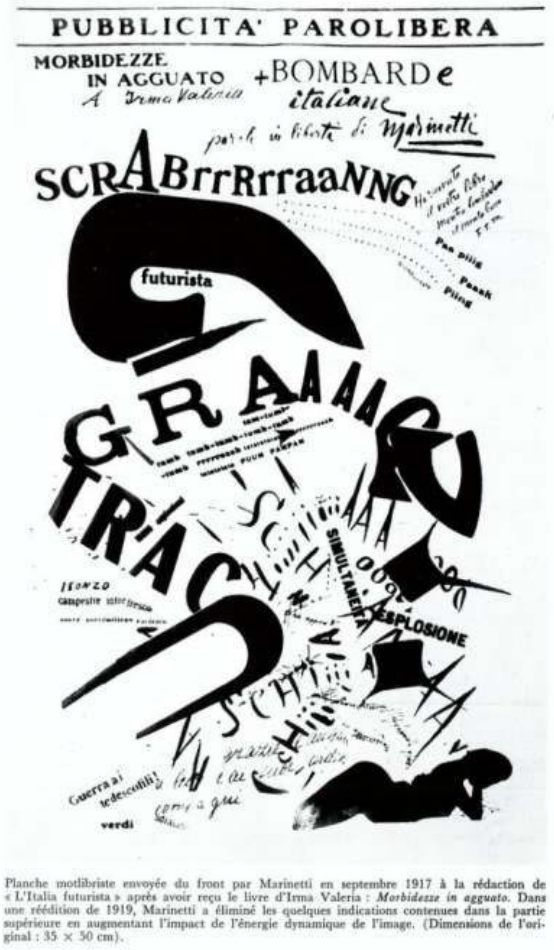

Reproduction in Giovanni Lista, Le livre futuriste, Modena, Panini, 1984

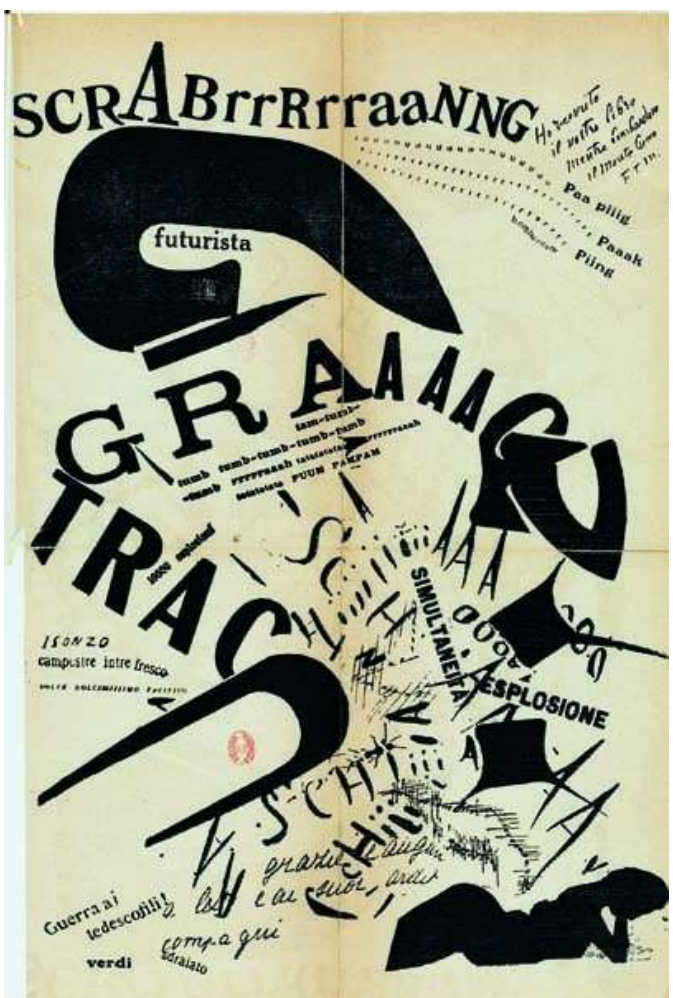

Reproduction in F.T. Marinetti, Les mots en liberté futuristes, Milano, Edizioni futuriste di «Poesia », $1919^{24}$

D'ailleurs il y a dans les carnets une sorte de mode d'emploi qui ressemble à la didascalie d'une pièce de théâtre; la déshumanisation des protagonistes renvoie évidemment aux 
«drames d'objets » conçus par Marinetti, Corra et Settimelli dans le théâtre synthétique futuriste d'avant la guerre ${ }^{25}$.

Décrire la fièvre charnelle transmise au papier à lettres par l'homme dans les tranchées et par la femme dans son lit à Paris. Avec le papier de la lettre encadrer chaque page de tous les bruits de la guerre et des bruits de Paris [...].

Il vaut mieux faire un rez-de-chaussée à la page avec les bruits de la guerre en les séparant bien les uns des autres, de sorte que l'on puisse oublier de temps en temps de lire les bruits. Comme celui qui absorbé dans la lecture d'un journal ou d'une lettre traverse une route ou sur un balcon et n'entend pas les bruits ${ }^{26}$.

Après avoir contextualisé la scène et posé le cadre, l'auteur se suggère l'opportunité de décrire le départ des deux lettres qui sont censées se croiser et qui, dès qu'elles sont à bord du train, se mettent à produire une espèce d'énergie cinétique capable de réchauffer les wagons. Après la didascalie en italien, la narration passe par la langue française :

C'est un train de blessés et de malades. Le wagon poste flambe ! enorme [sic] torche dans le paysage de boue infame [sic] sous l'entrecroisement des reflecteurs [sic]. Autour du wagon qui flambe ronde effrénée diaboliquement gaie de tous les blessés soudain guéris et de tous les malades sur pied.

Les lettres, protagonistes thaumaturges du récit, disparaissent tout à coup de la scène. Reste ce que Rouveyre avait appelé l'«érotisme imaginatif ${ }^{27}$ " des soldats blessés, soudainement guéris par la chaleur sensuelle qui se dégage des sacs de la Poste :

Ah! Ah! Des femmes! Des femmes! Des femmes! Du sexe!

Tous vont flairer et fourrer le nez et l'œil aux fentes des wagons qui sent le sexe.

Tous rient, rient !

Plus loin, Marinetti imagine un roman dont les protagonistes seraient les quarante-sept lettres de deux amants et les enveloppes qui les contiennent. Les écritures souhaiteraient ici tout à coup « rompre le carré du papier » (rompere il quadrato della carta). Comment ne pas saisir ici une écriture de l'Imaginaire qui, une fois de plus, s'organise autour de schèmes de l'agrandissement, de la multiplication, dans la tentative - vaine, évidemment - de dépasser les limites et les contours de l'espace et du temps en même temps qu'elle se bâtit des contours très précis?

L'« érotisme imaginatif » caractérise une partie importante du journal de Marinetti, ainsi qu'une partie prépondérante de ses projets romanesques autofictionnels. Presque toujours le protagoniste de ces récits s'exprime à la première personne du singulier et vante des performances sexuelles exceptionnelles: c'est le cas notamment dans «La Reine Bordel ", où il est capable de satisfaire les ardeurs de plusieurs femmes en pratiquant des cunnilingus à répétition :

Elles passent et l'une après l'autre elles soulèvent une jambe et prennent mon visage entre leurs cuisses. Je lèche furieusement chacune vibre de plaisir en déclarant avec un ton sérieux oh! Il ne se réveillera pas. Pendant que l'une se fait lécher l'autre pose un abat-jour identique sur la bougie de mon sexe et elle se le frotte contre sa chatte. La fille brune de Stanny murmure en passant à côté de moi qu'elle veut se le faire entrer dans le cul et que je jouisse ainsi. Le jeu finit ainsi. (La Reine Bordel $\left.{ }^{28}\right)$

31 L'idée - le fantasme - de l'homme capable de donner du plaisir à plusieurs femmes à la fois revient dans d'autres projets aux titres parfois coquins, «Le membre de mon mari défunt (veuve jeune et blonde)» (Il membro del mio post marito [vedova giovane bionda]), "L'opération chirurgicale " ('operazione chirurgica), et aux contenus pornographiques. Le niveau est souvent affligeant: dans «La récolte des fraises" (La raccolta delle fragole), Marinetti imagine un homme s'exprimant - comme toujours à la première personne du 
singulier, qui doit dénicher les fraises que deux femmes nues ont cachées sous leurs aisselles. Sans utiliser la force musculaire et en leur donnant du plaisir, il doit parvenir à leur faire lever les bras: «Je trouve les fraises et je vais les manger dans la chatte des deux femmes qui les avaient ${ }^{29}$.»

Vraisemblablement l'auteur Marinetti a été capable de faire le tri de ses écrits et, si la plupart d'entre eux sont restés inédits, d'autres, en revanche, ont été publiés à maintes reprises et avec très peu de variantes d'une version à l'autre: c'est le cas de «Il rasoio voluttuoso ", qui sera repris dans Gli amori futuristi, puis dans Il fauno galante sous le titre de "L'amorosa barbiera $»^{30}$, ainsi que dans Novelle colle labbra tinte ${ }^{31}$.

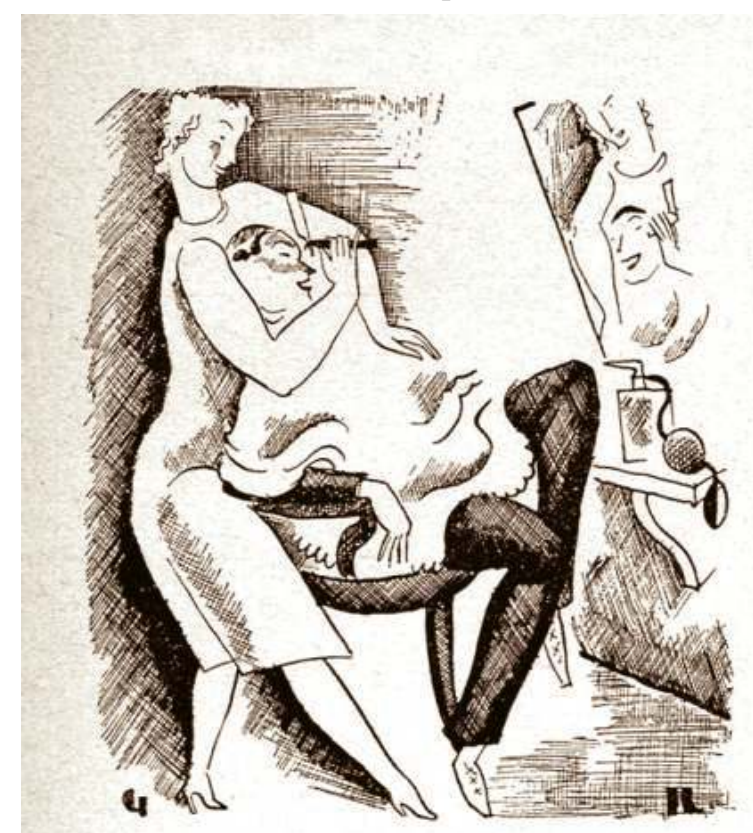

F. T. MARINETTI

L'AMOROSA BARBIERA

Reproduction de Giulio Rosso in Il fauno galante, "L'amorosa barbiera » ed altre molte storielle maliziose ${ }^{32}$

\section{Le roman est mort ; vive le roman !}

Ce qu'il convient de retenir, c'est que les délires imaginatifs de Marinetti et les projets conçus pendant la guerre - pour la plupart absents de l'édition des Taccuini-, quel que soit leur niveau, ne peuvent être analysés que comme une réponse à l'immanence dégradante - de la guerre. Son oscillation entre la récusation conceptuelle du genre romanesque et la pratique pourtant diffuse de l'écriture en prose ne peut être seulement la conséquence d'une stratégie de diffusion du futurisme. Autrement dit, l'exemple d'Umberto Notari - ami personnel de Marinetti et prolifique auteur de romans à scandale - et la situation éditoriale italienne ont sans doute contribué à pousser Marinetti dans la direction d'une pratique de l'écriture romanesque quelque peu licencieuse ${ }^{33}$. Toutefois, dans ces carnets où Marinetti, tel un Arioste, parle abondamment de femmes d'armes, d'amours et d'entreprises audacieuses, une véritable poétique est en train de se mettre en place dont seule une relecture systématique des textes manuscrits pourra cependant nous donner l'exacte teneur. 


\section{qu'il apparaît dans l'édition d'A. Bertoni :}

24 mars

Au lit dans la réserve de Moraro. Bronchite.

Dans le roman L'Amant rapide ou L'Interventionniste décrire largement et de façon convaincante les nuits tourmentées des batailles lointaines, les nuits de luxure déchirées envahies ravagées par les hordes russes criantes, par les bombardements. La nuit du Ronta avec les trains de munitions qui montent montent vers la frontière et les nuits glaciales dans la boue des tranchées avec les torrents de corps féminins nus nus ouverts allumés liquéfiés par le plaisir.

Guerre luxure $e^{34}$.

Voici à présent la transcription du manuscrit tenant compte des sauts à la ligne :

24 mars)

au lit

dans la réserve de Moraro.

Bronchite.

Dans le roman L'Amant

rapide ou L'Interventionniste

décrire largement

et de façon convaincante

les nuits tourmentées

des batailles lointaines,

les nuits de luxure

déchirées envahies rava

-gées par les hordes russes

criantes, par les bombar-

dements.

La nuit du Ronta

avec les trains de munitions

qui montent montent

vers la frontière

et les nuits glaciales dans la

boue des tranchées

avec les torrents de corps

féminins nus nus

ouverts allumés liqué-

fiés par le plaisir.

Guerre luxure

Le manuscrit est éminemment plus riche que la transcription, et la ponctuation bien différente : le rythme y est plus serré, plus saccadé, l'écriture plus saisissante, les sauts à la ligne toujours significatifs. Entre la description des «nuits de luxure» et des «nuits gelées dans la boue » il y a une coupure qui doit être lue comme une déchirure : la phrase continue à la ligne, sans majuscule, et sans avoir plus rien à voir avec le projet romanesque de description des «batailles lointaines». Tout à coup l'espace-temps se rétrécit et s'approche de l'ici, hic et nunc; « guerre » et « luxure » ne font plus qu'un. Cela ne signifie pas nécessairement que Marinetti jouit d'être au front: d'abord et avant tout nous sommes bien, ici, dans une sphère de création poïétique qui n'a plus rien à voir avec la contingence du présent. Ce qui jaillit de cette « guerre luxure », image au plus au point poiétique, est bien un monde nouveau, une réalité autre et sans doute éphémère, la 
victoire d'un fragment de poésie, le triomphe d'une écriture de l'Imaginaire qui se déploie, se répand par petits bouts, dépasse les contraires, du froid de la nuit à la chaleur des corps des femmes.

En somme : Marinetti ne peut pas renoncer à l'essence de sa poétique et à son identité de poète. Autrement dit, il est d'abord poète, avant que d'être soldat ou, tout au plus, il est un poète-soldat. Extrêmement prolifique et persuadé comme il l'est de la portée révolutionnaire du futurisme, il puisera abondamment dans ses notes manuscrites pour rédiger par la suite un certain nombre de manifestes, un roman - L'alcova d'acciaio-, quelques nouvelles. Il conviendra de revenir, dans le futur, sur les modalités de rédaction de ces textes manuscrits, en les répertoriant précisément et d'étudier les variantes; par ailleurs, il faudra poursuivre le travail d'analyse de la production romanesque de Marinetti, telle qu'elle commence à se dessiner dans les carnets : seul un travail d'édition des carnets pourra nous aider à trouver la réponse aux questions posées par la littérature narrative futuriste à partir de la deuxième moitié des années dix.

\section{NOTES}

1. "Je songe à un roman Lumière de femme ou Clair de femme. Après la guerre les forces manquent ", Taccuini : c'est ainsi que nous abrégerons dorénavant les citations tirées des carnets manuscrits de Marinetti conservés au fonds Marinetti à la Beinecke Library, Yale University. Nous avons pu consulter en microfilm la partie des carnets correspondant aux boîtes 41,42 et 43 $\mathrm{du}$ fonds Marinetti. Nous appellerons ces manuscrits Taccuini et, dans la mesure du possible, nous indiquerons le numéro du carnet tel qu'il apparaît dans le microfilm.

2. F. T. Marinetti, Taccuini. 1915-1921, Alberto Bertoni (a cura di), Bologna, Il Mulino, 1987 : il s'agit du volume que nous citons sous l'abréviation $T$.

3. Mina Della Pergola aurait approché le futurisme par la médiation de Bontempelli, dont elle était la belle-sœur: sous le pseudonyme de Diotima elle signe des textes parus dans L'Italia futurista. Actrice au Teatro degli Indipendenti dirigé par Bragaglia, elle a écrit des synthèses théâtrales. Cf. Futuriste. Letteratura. Arte. Vita, Giancarlo Carpi (a cura di), Roma, Castelvecchi, 2009, p. 620.

4. Enif Angiolini (1886-1974 ou 1976). Ses parents avaient choisi le prénom Enif en tirant au sort deux consonnes et deux voyelles, persuadés qu'il fallait que leurs enfants portent des prénoms à quatre lettres : c'est ainsi que la sœur d'Enif s'appelait Abes. Actrice, Enif et son mari, Alfredo Robert, faisaient partie de la troupe d'Eleonora Duse. En 1919 elle publie, avec Marinetti, le roman Un ventre di donna. Cf. Barbara Ballardin, Enif Angiolini Robert: futurista, amica di Marinetti, attrice, fedelissima della Duse, Milano, Selene, 2010. Cf. également nos deux travaux sur Enif Robert : «Enif Robert e Marinetti: l'autobiografia futurista a due voci », in Plurilinguisme et Avantgardes, Franca Bruera et Barbara Meazzi (éd.), Bruxelles, P.I.E. Peter Lang, 2011, p. 345-359, et Barbara Meazzi, « Enif Robert \& Filippo Tommaso Marinetti: Un ventre di donna e l'autobiografia futurista ", in Tempo e memoria nella lingua e nella letteratura italiana, Bart Van den Bossche et al. (a cura di), Bruxelles, AIPI, 2009, p. 19-41, http://www.infoaipi.org/attion/ascoli_vol_3.pdf.

5. Dans nos travaux sur Un ventre di donna (entre autres : "Enif Robert \& Filippo Tommaso Marinetti : Un ventre di donna e l'autobiografia futurista ", cit.), nous avons pensé que l'éditeur 
avait coupé des parties du texte par respect pour les héritiers. Il n'en est rien : pendant cette période Marinetti utilise du papier fin; l'encre est passée à travers et le texte résultant est très peu lisible.

6. « Enif Robert. Anima inquieta. Anima ultra nervosa. "Mi piace verniciarmi di cattiveria. Poi mi pento" ", T, 27 janvier 1918, p. 189.

7. «Enif Robert. Anima inquieta. Anima ultra nervosa. « Mi piace verniciarmi di cattiveria. Poi mi pento. Oggi non mi vuoi bene. Un'ombra è scesa fra di noi. Perché [ill.]» Enif mi dice queste parole strane [ill.] a letto [ill.]. »

8. Claudia Salaris avait déjà formulé cette hypothèse : cf. Claudia Salaris, Marinetti. Arte e vita futurista, Roma, Editori Riuniti, 1997, p. 184.

9. Taccuini, $1^{\mathrm{er}}$ carnet.

10. «Affinità / antiveggenza / effusioni di amorevolezza / ragrinzare (sic) la bocca per cominciare a piangere / ha ripugnanza di qualsiasi bassezza / soddisfare la sete / giacca panciotto / per le scale mi veniva dietro piano piano reggermi alla ringhiera / Ti ho veduto a braccetto con un ubriaco / lunga tavola intorno a cui sedevano tutti quegli uomini / caddi in ginocchio / camminando lungo il parapetto / una luce dolce rosea [...] continua senza fine ", Taccuini, $2^{\mathrm{e}}$ carnet, 3 mai. Marinetti fait un saut à la ligne après chaque mot ou phrase et nous avons signalé cela par la barre oblique. Nous avons raccourci le texte.

11. «Da che si fa buio, sto in ascolto / Soffriva di geloni / Piedi piagati sanguinanti / Allacciare le scarpe / Sentivo le sue mani appoggiate sulle mie spalle mentre le leccavo la fica in ginocchio / Io mi indugiavo e le sue mani tremavano / Quanti giorni gli date a giudicarlo dall'aspetto? / Sento sulla bocca l'impressione delle tue palpebre dolci come due viole / Andrò dritto alla fine / Dove eravamo rimasti? / A tavola gli stavo quasi di contro / Soverchiava tutti / Non soffriva contraddizioni / Repulsione e attrazione / Sulla fine del desinare / S'adirava facilmente / Teneva testa a tutti / Il sole, forse reso audace dal vino di tante taverne saccheggiate e dal sangue [ill.] teneva testa alla notte ", Taccuini, $10^{\mathrm{e}}$ carnet, 31 mars.

12. Nous avons consulté ces carnets en microfilm : comme le signale Domenico Cammarota dans la fiche bibliographique des Novelle colle labbra tinte, l'ordre des carnets - qui sont pourtant numérotés - n'est que théorique. Comme cela arrive quelquefois dans les archives, l'enthousiasme scientifique des chercheurs peut avoir comme conséquence la désorganisation des documents, ce qui n'émeut pas particulièrement des conservateurs soucieux de la préservation des documents mais peu attentifs aux contenus et à leur classement chronologique. Quoi qu'il en soit, nous exprimons une fois de plus notre infinie reconnaissance à la Beinecke Rare Book and Manuscript Library, à la Yale University, où sont conservés les carnets de Marinetti.

13. F. T. Marinetti, Parole in libertà futuriste tattili termiche olfattive, Roma, Edizioni futuriste di Poesia, 1932 ; Tullio D'Albisola, L'anguria lirica, Milano, Officine d'arti grafiche Chiattone, 1934.

14. F.T. Marinetti, Amanti simultanei - romanzo discato, 1938. Le manuscrit est conservé à The Getty Research Institute - Research Library

15. Par exemple Igino Ugo Tarchetti, «Idee minime sul romanzo », in Rivista minima, 31 octobre 1865, repris dans Igino Ugo Tarchetti, Tutte le opere, Enrico Ghidetti (a cura di), Bologna, Cappelli, 1967, vol. 2, p. 522 et suivantes.

16. « 6789 aprile [1918, n.d.r.] [...] Il romanzo deve morire. La sua nostalgia è ora fuori posto. Da Omero a Rosny e D'Annunzio il romanzo ha mantenuto sempre il suo ritmo nostalgico di racconto sconsolato: C'era una volta... Noi opponiamo a questo lamento-rimpianto: fà, scegli sfonda domina così così e anche così... così così! così! ", T, p. 220. Rosny était le pseudonyme commun des frères Boex, auteurs d'origine belge de romans fantastiques et de science-fiction, actifs dès la fin du xix ${ }^{\mathrm{e}}$ siècle. En 1909 ils cesseront d'écrire ensemble et choisiront de signer leurs romans respectifs comme J.-H. Rosny aîné et J.-H. Rosny jeune. Decio Cinti traduira pour Sonzogno un certain nombre de leurs romans et, entre autres, en 1922, La Guerre du feu. 
17. F.T. Marinetti, Gli amori futuristi, Cremona, Casa Editrice Ghelfi Costantino, 1922.

18. « [p]rogrammi di vita con ramificazioni. Cioè con varianti finali. 2 o 3 finali possibili diversi l'uno dall'altro e a scelta », T, p. 207.

19. F.T. Marinetti, L'alcova d'acciaio, Milano, Vitagliano, 1921.

20. «Io, qui, non ho altro che del fango... Ma, come Dio col fango plasmò l'uomo, io spero di plasmare il nuovo mondo futurista italiano", Enif Robert, F.T. Marinetti, Un ventre di donna, Milano, Facchi, 1919, p. 296.

21. «Organizzo il mio romanzo L'interventista o Contro il deposito del chiaro di luna o L'amante veloce. [Frenesia di treni da Milano a Roma Napoli. Dimostrazione intervento al Dal Verme in Galleria. 8 bandiere austriache bruciate. Brevi soste a Rapallo Golfo di dolcezza erotica a gambe aperte.] Un altro romanzo: La lettera carnale o La lettera di carne. (21 marzo 1917)»T, p. 64. La partie entre parenthèses est inédite.

22. "A letto alla riserva di Moraro. Bronchite. Nel romanzo L'amante veloce o L'interventista descrivere ampiamente e persuasivamente le notti tormentate delle battaglie lontane, le notti di lussuria dilaniate invase devastate dalle orde russe urlanti, dai bombardamenti. (24 marzo $1917) » T$, p. 65. Nous n'avons pas compris pourquoi les hordes seraient russes, mais c'est bien ce qu'il note dans son texte.

23. «Voglio scrivere parole in libertà / Il paesaggio d'odori del mio cane / Gli odori della mia Amica / Dare un coito appassionato con parole in libertà. / (Titoli di libri Lussuria di guerra Le mie notti La signora [ill.] Pagine roventi Notti roventi Letto campo di battaglia Sacco a pelo I problemi del mio corpo Bordello di guerra vicino a trincee.) (18 aprile 1918)», T, p. 221. La partie entre parenthèses est inédite.

24. La planche de gauche [Ndlr: planche du haut] a été reproduite, entre autres, in Giovanni Lista, Le livre futuriste, Modena, Panini, 1984 ; la planche de droite [Ndlr: planche du bas] est reproduite dans F.T. Marinetti, Les mots en liberté futuristes, Milano, Edizioni futuriste di « Poesia », 1919

25. Cf. par exemple «Vengono»: F. T. Marinetti, Teatro, Giovanni Calendoli (a cura di), vol. II, Roma, Vito Bianco Edizioni, 1969, p. 283 et suivantes.

26. «Dare la febbre carnale trasmessa alla carta da lui in trincea e da lei nel suo letto a Parigi. Colla carta della lettera incorniciare ogni pagina di tutti rumori di guerra e dai rumori parigini [...].

Meglio fare un rez de chaussé [sic] alla pagina coi rumori di guerra separando bene l'uno dall'altro per modo che si possa dimenticare di tanto in tanto di leggere i rumori. Come colui che assorto in una lettura di giornale o lettera traversa una strada o sta al balcone senza sentire i rumori ». La partie que nous publions ici n'a pas été transcrite et suit la phrase «Un romanzo Deux lettres d'amour se rencontrent ", T, p. 69.

27. Cf. Barbara Meazzi, "C'est la guéguerre! Apollinaire et Marinetti à la guerre comme à l'amour ", in L'Écriture en guerre de Guillaume Apollinaire, cit., p. 209-226.

28. « [...] Passano e una dopo l'altra alzano una gamba prendendomi la faccia tra le cosce. Io lecco furiosamente ognuna vibra dal piacere dichiarando con tono serio Oh! Il ne se reveillera [sic] pas. [mots cassés] Mentre l'una si fa leccare l'altra mette un identico abat-jour sulla candela del mio membro e se lo [mots cassés] frega contro la fica. La bruna figlia di Stanny mi sussurra passando che vuole farselo entrare nel culo e che io goda così. Il gioco finisce così. (La Reine Bordel) ", Taccuini, $2^{\mathrm{e}}$ carnet.

29. " Trovo le fragole e vado a mangiarmele nella fica delle due donne che le avevano », Taccuini, $2^{\mathrm{e}}$ carnet.

30. Il fauno galante, «L'amorosa barbiera » ed altre molte storielle maliziose, vol. I, Roma, Ed. D'Arte Fauno, 1927.

31. F.T. Marinetti, Novelle colle labbra tinte, Milano, Mondadori, 1930.

32. Nous reproduisons ici le dessin de Giulio Rosso, illustrateur du recueil Il fauno galante, "L'amorosa barbiera » ed altre molte storielle maliziose, cit. 
33. Cette même stratégie sera utilisée par Benedetta dans les années cinquante, afin de commencer la réhabilitation du futurisme et de Marinetti en Italie: nous avons trouvé un numéro de Le Ore, une revue vaguement « nostalgico-fasciste » et érotique - du moins lors de son lancement en 1953, car plus tard ce sera une revue pornographique - où, entre une photo de l'actrice Rita Giannuzzi montrant un décolleté généreux en couverture, la commémoration de Marcinelle et la mode du tatouage, on raconte l'histoire illustrée du futurisme. "Benedetta è l'ombra incarnata di Marinetti, nume tutelare, sposa e sorella e, oggi, difesa assoluta, antenna prestigiosa che raccoglie tutte le voci del mondo e le trasmette al suo Marinetti " [Benedetta est l'ombre incarnée de Marinetti, divinité tutélaire, épouse et sœur et, aujourd'hui, défense absolue, antenne prestigieuse rassemblant toutes les voix du monde et les envoyant à son Marinetti]. Dans l'article, peu précis - Benedetta aurait rencontré Marinetti chez Ignazio (sic) Balla , on dit que Marinetti a laissé onze ouvrages inédits à une veuve vivant dans le culte du mari et complètement vouée à la mémoire du futurisme. Bien entendu, aucune allusion n'est faite au passé fasciste de Marinetti. Marcello Gallian, «Storia illustrata di Marinetti e dei suoi. Il futuro del passato », Le Ore, ${ }^{\circ} 171$, 18 août 1956, p. 38-42. Dans le numéro suivant (Le Ore, $\mathrm{n}^{\circ}$ 172, 25 août 1956, p. 33-37), entre une photo de Marisa Allasio en short et un portrait de Michèle Morgan en vacances, Gallian parle de théâtre et de peinture futuristes. Marcello Gallian, écrivain, journaliste et peintre anarchofasciste, avait fait partie du groupe 900.

34. « 24 marzo / A letto alla riserva di Moraro. Bronchite. / Nel romanzo L'amante veloce o L'interventista descrivere ampiamente e persuasivamente le notti tormentate delle battaglie lontane, le notti di lussuria dilaniate invase devastate dalle orde russe urlanti, dai bombardamenti. / La notte di Ronta coi treni di munizioni che salgono salgono verso la frontiera e le notti gelate nel fango della trincea coi torrenti di corpi femminili nudi nudi aperti accesi liquefatti dal piacere. / Guerra lussuria. », T, p. 65.

\section{RÉSUMÉS}

Le futurisme a eu un rapport paradoxal avec le roman : le genre est officiellement déconsidéré - un seul manifeste officiellement consacré au roman (synthétique) paraîtra en 1939-, pourtant les futuristes et Marinetti pratiquent l'écriture romanesque sans trop se soucier d'une quelconque cohérence. Et même, dès les années de la guerre, Marinetti - au front - ne cesse d'imaginer des ébauches romanesques de toutes sortes, qu'il note précisément dans ses carnets manuscrits partiellement publiés il y a une trentaine d'années.

Dans notre travail, nous analysons les spécificités de certains de ces innombrables projets narratifs, dont nous avons vérifié la précision de la transcription par rapport à la version manuscrite : il ressort ainsi que la version originale est bien plus riche, non seulement parce que les projets romanesques sont souvent explicités, mais aussi parce que, à bien des égards et audelà des intentions, certains passages peuvent être lus comme de véritables textes poétiques.

Il futurismo ebbe un rapporto paradossale con il romanzo : il genere è stato poco considerato - un solo manifesto sarà ufficialmente consacrato al romanzo (sintetico) nel 1939-, eppure i futuristi e Marinetti praticano la scrittura romanzesca senza preoccuparsi della coerenza. Lo stesso Marinetti, fin dagli anni della guerra, mentre è al fronte immagina innumerevoli nuclei narrativi di vario tipo, che annota con grande precisione sui taccuini manoscritti parzialmente pubblicati una trentina di anni fa. 
Nel nostro lavoro, abbiamo analizzato le specificità di alcuni di questi numerosissimi progetti narrativi, di cui abbiamo verificato la precisione della trascrizione, rispetto alla versione manoscritta: la versione originale risulta ben più ricca non solo perché i progetti romanzeschi vengono spesso esplicitati, ma anche perché, a tutti gli effetti e al di là delle intenzioni, alcuni passaggi possono essere letti come dei veri e propri testi poetici.

\section{INDEX}

Index géographique : Italie, France

Mots-clés : futurisme, roman, récit, manuscrit, imaginaire

Index chronologique : années 1910

\section{AUTEUR}

\section{BARBARA MEAZZI}

Université Nice Sophia Antipolis

Barbara Meazzi est Professeure d'Italien à l'Université Nice Sophia Antipolis et a travaillé auparavant à l'Université de Savoie. Elle travaille sur le futurisme depuis sa thèse de doctorat et elle a d'ailleurs consacré un travail sur Le futurisme entre l'Italie et la France - 1909-1919, paru aux Presses de l'Université de Savoie. Elle a co-dirigé trois ouvrages, Les oubliés des avant-gardes (2006), Une traversée du XX siècle : arts, littérature, philosophie. Hommage à Jean Burgos (2008), et Plurilinguisme et Avant-gardes (2010), avec Franca Bruera, paru chez Peter Lang. En 2011 elle a également publié une anthologie de textes futuristes sur l'art de la séduction, L'arte futurista di piacere. Sintesi di tecniche di seduzione, parue chez Nerosubianco Edizioni en Italie.

Elle a édité la correspondance inédite d'Ardengo Soffici avec Serge Férat et Hélène d'Ætttingen, dont la publication est prévue chez L’Âge d'Homme pour septembre 2013 ; elle prépare actuellement un volume sur la prose futuriste des années dix et vingt. 